\title{
Imaging three-dimensional rotational diffusion of plasmon resonant gold nanorods using polarization-sensitive optical coherence tomography
}

\author{
Raghav K. Chhetri ${ }^{1}$, Krystian A. Kozek ${ }^{2}$, Aaron C. Johnston-Peck ${ }^{2}$, Joseph B. Tracy ${ }^{2}$, and \\ Amy L. Oldenburg ${ }^{1,3,{ }^{*}}$ \\ ${ }^{1}$ Department of Physics and Astronomy, University of North Carolina at Chapel Hill, Chapel Hill, \\ North Carolina 27599, USA \\ ${ }^{2}$ Department of Materials Science and Engineering, North Carolina State University, Raleigh, \\ North Carolina 27695, USA \\ ${ }^{3}$ Biomedical Research Imaging Center, University of North Carolina at Chapel Hill, Chapel Hill, \\ North Carolina 27599, USA
}

\begin{abstract}
We demonstrate depth-resolved viscosity measurements within a single object using polarized optical scattering from ensembles of freely tumbling plasmon resonant gold nanorods (GNRs) monitored with polarization-sensitive optical coherence tomography. The rotational diffusion coefficient of the GNRs is shown to correlate with viscosity in molecular fluids according to the Stokes-Einstein relation. The plasmon resonant and highly anisotropic properties of GNRs are favorable for microrheological studies of nanoscale properties.
\end{abstract}

In the growing field of microrheology, there has been considerable interest in techniques that quantify thermal diffusion of probes within locally resolved volumes of the medium under investigation. The Stokes-Einstein relation relates diffusion measured by these passive techniques to the linear viscoelastic properties of the medium, provided that the probe is inert and the medium behaves as a near-equilibrium, homogeneous, isotropic, and incompressible continuum [1]. Optical coherence tomography (OCT) [2] provides a novel platform to study dynamic light scattering (DLS) from passively diffusing particles [3]. Using plasmon resonant gold nanorods (GNRs), DLS with OCT enables locally resolved, passive microrheology of medium properties with microscale heterogeneities.

We investigated polarized light scattering from ensembles of GNRs using polarizationsensitive OCT (PS-OCT) to depth resolve the rotational diffusion coefficient $\left(D_{R}\right)$ in media of varying viscosity. The shape anisotropy of a GNR splits the surface plasmon resonance into transverse and longitudinal modes, the latter of which provides high light scattering efficiencies due to reduced plasmon damping, and is polarized parallel to the long axis of the GNR [4]. By monitoring the polarized scattering, it has previously been shown that a GNR under two-dimensional (2D) Brownian rotation can be used as a local orientation sensor [5]. We expect GNRs in the molecular fluids in our study to obey the Stokes-Einstein relation, so that their three-dimensional (3D) Brownian diffusion can be related to the viscosity of the medium. While the translational diffusion of nanorods is complicated by coupling to rotational diffusion due to the shape anisotropy [6], rotational diffusion is independent of the

C2011 American Physical Society

*Author to whom correspondence should be addressed: aold@physics.unc.edu. 
state of translation, and as such, is a robust metric for local viscous properties. Furthermore, we expect GNRs to probe the viscosity of the medium at a smaller scale than that possible by using traditional microparticles.

In this study we employ OCT to monitor $D_{R}$ of ensembles of unconfined GNRs. OCT employs optical depth ranging of singly backscattered light, enabling real-time imaging in noninvasive biomedical applications. Similar to DLS techniques, OCT is an optical heterodyne method which senses ensemble-averaged scattering from scatterers within the coherence volume, providing a higher signal-to-noise ratio and speed compared to singleparticle tracking methods. Unlike traditional DLS, OCT employs low-coherence light so that the coherence volume is small; as such, OCT is capable of resolving the dynamic signal from each local coherence volume over depths exceeding the mean scattering path length [7], which has implications for analyzing optically thick tissues. The localized-coherencevolume technique has previously been used with microbeads to perform spatially resolved microrheology [8].

In this Rapid Communication, we employ PS-OCT and principles from DLS to measure the $D_{R}$ of ensembles of GNRs freely suspended in media of varying viscosity. We test the validity of the Stokes-Einstein relation by comparing the observed $D_{R}$ values with a model for the Stokes drag on cylinders, modified to account for the non-negligible GNR size distribution by computing the temporal statistics of a representative ensemble of GNRs. Using these validation measurements relating $D_{R}$ to the viscosity of the medium, we demonstrate the capability of PS-OCT to spatially map the viscosity of a heterogeneous sample by using GNRs as rheological probes. The ability to resolve micrometer-scale heterogeneities in viscosity using GNRs with OCT may open new avenues for microrheological investigation.

GNRs used in this study have an average length and width of $53 \pm 10$ and $15 \pm 4 \mathrm{~nm}$, respectively, and exhibit a strong longitudinal plasmon mode centered at $780 \mathrm{~nm}$ with a full width at half maximum of $140 \mathrm{~nm}$, which is within the OCT source spectrum that spans $735-865 \mathrm{~nm}$ at half maximum [9]. Two batch solutions for the experiment were prepared by mixing $10 \%$ of the GNRs solution $\left(\sim 8 \times 10^{8} \mathrm{GNRs} / \mu \mathrm{L}\right)$ with glycerol (Acros Organics, 15892-0010) and water (Fisher Scientific, W5-4), respectively. Samples with varying viscosity were then prepared by mixing the two batch solutions in different proportions, and their resulting viscosities were estimated using a mixture law [10]. Although multiple GNRs populate each coherence volume, we expect them to be noninteracting, because the estimated average separation between the GNRs (minimum $\sim 1450 \mathrm{~nm}$ ) is large compared to both the average length of a GNR $(\sim 53 \mathrm{~nm})$ and the mean distance the GNRs travel over the duration of the OCT measurement (maximum $\sim 140 \mathrm{~nm}$ ).

The OCT system in this study is a spectral domain, polarization-sensitive system, as shown in Fig. 1(a). The light source consists of a Ti:sapphire laser (Griffin, KMLabs, Inc.) and provides a coherence gate of $\sim 2.6 \mu \mathrm{m}$ in air. Light from the source is horizontally polarized and split into reference and sample arms. Imaging is performed by a lens $(f=30 \mathrm{~mm})$, which provides a transverse resolution of $\sim 12 \mu \mathrm{m}$ in air. Owing to the coherence gate and the transverse resolution, the coherence volume is estimated to be $\sim 375 \mu \mathrm{m}^{3}$ in air. Horizontally polarized light $(\sim 5 \mathrm{~mW})$ incident upon the sample is backscattered into both horizontal $(H)$ and vertical $(V)$ polarization states, which interfere with their respective polarization states from the reference (consisting of linearly polarized light at $45^{\circ}$, generated after double pass through a quarter-wave plate at $22.5^{\circ}$ ). The interfered light is split into horizontal and vertical components by a polarizing beam splitter $(H H$ and $H V$, respectively, where the first and second terms are the incident and backscattered polarization states from the sample, respectively), and directed to a custom spectrometer. The spectrometer is similar to a 
previous design [11], and consists of a transmission grating (600 lines $/ \mathrm{mm}$, Wasatch Photonics), camera lens $(f=200 \mathrm{~mm}$ ), and a line scan camera (Piranha, Dalsa Inc.) operated at $25 \mathrm{kHz}$, providing an imaging depth of $2.08 \mathrm{~mm}$ in air.

Dynamic PS-OCT signals were recorded by acquiring depth scans from the same transverse position in the sample as a function of time ( $M$ mode). A total of 4000 depth scans were obtained with a line rate of $25 \mathrm{kHz}$ (i.e., sampled every $40 \mu \mathrm{s}$ for an overall duration of 160 $\mathrm{ms})$. Typical $M$-mode OCT images showing time traces of the depth-resolved intensity fluctuations for samples with different viscosities are shown in Fig. 1(b). Qualitatively, we observe that the intensity fluctuations along the horizontal (time) axis are much slower in a high viscosity sample than in a low viscosity sample. Quantitative analysis, as performed below, reveals that the time scale of these intensity fluctuations is directly related to the viscosity of the medium.

Spectral domain OCT is a heterodyne detection scheme in which the complex analytic signal $\tilde{a}(z, t)$ as a function of depth $z$ in the sample is obtained by inverse Fourier transformation of the measured spectrum [12]. In the heterodyne experiment, the temporal autocorrelation of the real part of $\tilde{a}(z, t), G^{(2)}(z, \tau)$, is relatable to the first-order correlation function of the electric field scattered from the sample, $G^{(1)}(z, \tau)$ [13]. In this study, we employ polarizationsensitive OCT to collect the cross-polarized $(H V)$ dynamic light scattering signal because it provides direct access to $D_{R}$ against a background of slow translational diffusion $D_{T}$. Specifically, (after normalization),

$$
g_{H V}^{(1)}(z, \tau)=e^{-\left[6 D_{R}(z)+q^{2} D_{T}(z)\right] \tau} \approx e^{-6 D_{R}(z) \tau},
$$

where $q=4 \pi n / \lambda_{0}$ is the scattering vector in the backscattering geometry, the fast $e^{-i \omega_{0} \tau}$ term is dropped for convenience, and $6 D_{R} \gg q^{2} D_{T}$ for the GNRs under study (by a factor of $\sim 38$ for an average GNR of length $53 \mathrm{~nm}$ and width $15 \mathrm{~nm}$, using expressions previously reported [14]). Therefore, $D_{R}$ equates to $\left(6 \tau_{1 / e}\right)^{-1}$, where $\tau_{1 / e}$ is the $1 / e$ decay time of $g_{H V}^{(1)}$.

Computationally, $D_{R}$ at each depth $z$ was isolated from $\tilde{a}_{H V}(z, t)$ as follows: The real part of $\tilde{a}_{H V}(z, t)$ was taken, and the time average was then subtracted to control for nonzero background noise and to isolate the intensity fluctuation [15]. Then, the autocorrelations were evaluated at each $z$ and normalized to obtain $g_{H V}^{(1)}$, averaged within multiple depth intervals ( $N=7$, with each depth interval chosen to be $35 \mu \mathrm{m}$ ), and fitted to the expected inverse exponential of Eq. (1). A representative $g_{H V}^{(1)}$ for each sample is shown in Fig. 2, with a sample inverse-exponential fitting shown in the inset. The fittings were performed over a windowed region of $g_{H V}^{(1)}$ from $\tau=0$ to $\sim \tau_{1 / e}$. We find that the measured $g_{H V}^{(1)}$ values appear to be consistently larger than those of a pure exponential at times greater than $\tau_{1 / e}$. This may be explained partially by the size distribution of GNRs giving rise to a distribution of rotational rates [as modeled in Eq. (2)], which deviates from a pure exponential in qualitatively the same manner, or it may be explained partially by the translational diffusion of the GNRs becoming more significant at longer time scales. The rotational diffusion rate was then calculated at each depth interval according to $D_{R}=\left(6 \tau_{1 / e}\right)^{-1}$. We noted that there was no significant change in $D_{R}$ versus depth, which was expected because the mean scattering path length from GNRs is much longer than the depths analyzed. The $D_{R}$ values averaged over multiple depth intervals are plotted in Fig. 3. As expected, an inverse relationship between $D_{R}$ and viscosity is found, which suggests that the rotations of the GNRs occur over a comparatively shorter time scale as the viscosity decreases. We noted that $D_{R}$ values were consistent (within 7\%)when the concentration of the GNRs was decreased from 10\% (at 
which the experiment was performed) to $2 \%$, while maintaining the viscosity at a constant value (within 5\%). Given the consistency of $D_{R}$ with concentration and the invariance of measured $D_{R}$ with depth, the effect of multiple scattering is believed to be negligible in our experiment.

To test the validity of the Stokes-Einstein relation for this system, the experimental $D_{R}$ values were compared with a model combining the rotational diffusion of smooth cylinders [14] with the optical scattering from ellipsoids [16] while accounting for the measured size distribution of the GNRs. First, simulated autocorrelations were computed as the sum of the contribution of each GNR in a representative distribution $(n=998)$ as follows:

$$
g_{H V}^{(1)}(\tau)=\sum_{j=1}^{998} c_{j}^{2} e^{-6 D_{R j} \tau},
$$

where the summation is over each nanorod $j$ in the distribution, and $c_{j}^{2}$ is an optical weighting factor accounting for the maximum fluctuation in backscattering detected by the OCT system from each GNR. This is important to overcome the experimental bias for more efficient detection of larger, more slowly diffusing GNRs. The weights $c_{j}^{2}$ were evaluated from the optical scattering anisotropy of each GNR weighted by the incident light spectrum, according to

$$
c_{j}^{2}=\sum_{\lambda}\left[\sigma_{s, \|}\left(\lambda, L_{j}, d_{j}\right)-\sigma_{s, \perp}\left(\lambda, L_{j}, d_{j}\right)\right] E_{r}^{2}(\lambda)
$$

with $L_{j}$ and $d_{j}$ the length and width of the $j$ th GNR, and $\sigma_{s, \|}$ and $\sigma_{s, \perp \text {; }}$ the scattering cross sections, computed according to Mie Gans theory [16], of the jth GNR when the incident polarization is parallel and perpendicular to the long axis, respectively. On average, we find that $\sigma_{s, \|}$ is $\sim 250$ times that of $\sigma_{s, \perp}$, highlighting the high scattering anisotropy exhibited by GNRs. To compute $D_{R j}$ in Eq. (2) for each GNR, an expression derived for solid cylinders $(2<L / d<20)$ was employed [14]. Theoretical $D_{R}$ were then evaluated by fitting an inverse exponential of the form shown in Eq. (1) to the simulated autocorrelation given by Eq. (2).

Theoretical predictions were made in two ways: first, by considering the actual sizes of the GNRs measured by transmission electron microscopy (TEM), and second, by considering an average surfactant [cetyltrimethylammonium bromide (CTAB)] capping thickness of $2 \mathrm{~nm}$ around the GNRs. The average CTAB layer thickness was estimated from a TEM image as half of the average spacing between the GNRs when densely packed, and is consistent with previously reported values for similarly sized GNRs, synthesized using a growth method in the presence of CTAB [17].

We find that the experimental $D_{R}$ values correlate with the theoretical $D_{R}$ computed as above for our distribution of GNRs (Fig. 3). Agreement between experimental and theoretical $D_{R}$ is obtained over a viscosity range of 42-249 mPas, and the level of agreement with theory is similar to the findings of a DLS study of similarly sized GNRs at a single viscosity [18]. In making direct comparisons between the experimental values and the theoretical predictions, it should be taken into consideration that the theoretical model is only an approximation; the GNRs are not exactly cylindrical in shape (as assumed for the rotational diffusion model), nor are they exactly ellipsoidal (as assumed for the optical scattering model). 
These results demonstrate that PS-OCT can monitor viscosity using GNRs as nanoprobes within the applicability of the Stokes-Einstein relation. Next, the potential of our technique to resolve heterogeneities in viscosity was explored using a double chamber, as shown in Fig. 4(a), in which two samples with different viscosities were separated by a thin microscope cover glass. Figure 4(b) shows the $M$-mode images of the two samples in the double chamber. Figure 4(c) shows $g_{H V}^{(1)}$ averaged within each chamber, demonstrating two different time scales that indicate the difference in viscosity in the two chambers. By fitting

$g_{H V}^{(1)}$ to the expected inverse exponential of Eq. (1), $D_{R}$ of $72 \pm 5$ and $227 \pm 6 \mathrm{~s}^{-1}$ were found for samples in the top and bottom chamber, respectively, which are consistent with independent measurements of $D_{R}$ of $74 \pm 5$ and $246 \pm 13 \mathrm{~s}^{-1}$, respectively, for these samples (Fig. 2). Figure 4(d) shows $D_{R}$ as a function of depth in the double chamber, where $D_{R}$ was computed within each depth interval of $35 \mu \mathrm{m}$, and the depth intervals were successively stepped by $14 \mu \mathrm{m}$ through both chambers. A clear distinction between the rotational diffusion coefficients of the two samples is seen, and the $D_{R}$ values measured within multiple depth intervals for the same sample are also consistent with one another. This demonstrates that DLS performed with PS-OCT using GNRs as nanoprobes is capable of resolving the microscale heterogeneities in viscosity existing at multiple depths within an object.

In conclusion, by combining the techniques of PS-OCT and DLS, we showed that polarization-dependent scattering from ensembles of GNRs provides information about $D_{R}$, and subsequently the viscosity of the medium. We have studied the unconfined stochastic motion of plasmon resonant GNRs in 3D using OCT, and obtained agreement between experimental and theoretical $D_{R}$ over a viscosity range of $42-249 \mathrm{mPas}$, which is within the regime of interest in biophysical studies. Averaging over large numbers of GNRs provides a high signal-to-noise ratio for determining the viscosity within each coherence volume of the sample (in this study, $\sim 130$ GNRs within each coherence volume of $\sim 375 \mu \mathrm{m}^{3}$ ). We also demonstrated the ability to depth resolve the heterogeneous viscosity within a single object using this technique. Future work is needed to validate this technique in non-Newtonian fluids using the generalized Stokes-Einstein relation. Because OCT rejects multiply scattered light, this technique has the potential to provide microrheology in optically thick samples, such as biological tissues. The combination of the anisotropic and plasmon resonant properties of GNRs with OCT provides a unique tool for imaging microscale heterogeneities of rheological properties.

\section{Supplementary Material}

Refer to Web version on PubMed Central for supplementary material.

\section{Acknowledgments}

This work was supported by a pilot grant from the Carolina Cancer Center for Nanotechnology Excellence [CCCNE NIH (NCI) No. U54CA119343] and by startup funds at UNC-Chapel Hill and NCSU. A.C.J.-.P acknowledges support from GAANN.

\section{References}

1. Squires TM, Mason Thomas G. Annu. Rev. Fluid Mech. 2010; 42:413.

2. Huang D, et al. Science. 1991; 254:1178. [PubMed: 1957169]

3. Kalkman J, Sprik R, van Leeuwen TG. Phys. Rev. Lett. 2010; 105:198302. [PubMed: 21231201]

4. Sönnichsen C, et al. Phys. Rev. Lett. 2002; 88:077402. [PubMed: 11863939]

5. Sönnichsen C, Alivisatos AP. Nano Lett. 2005; 5:301. [PubMed: 15794615]

6. Han Y, et al. Science. 2006; 314:626. [PubMed: 17068256] 
7. Boas DA, Bizheva KK, Siegel AM. Opt. Lett. 1998; 23:319. [PubMed: 18084498]

8. Popescu G, Dogariu A, Rajagopalan R. Phys. Rev. E. 2002; 65:041504.

9. See supplemental material at [http://link.aps.org/supplemental/10.1103/PhysRevE.83.040903] for the synthesis and absorbance spectrum.

10. Cheng N-S. Ind. Eng. Chem. Res. 2008; 47:3285.

11. Baumann B, et al. Opt. Express. 2007; 15:1054. [PubMed: 19532333]

12. Fercher AF, et al. Opt. Commun. 1995; 117:43.

13. Johnson, CS., Jr; Gabriel, DA. Laser Light Scattering. New York: Dover; 1994.

14. Ortega A, García de la Torre J. J. Chem. Phys. 2003; 119:9914.

15. Berne, BJ.; Pecora, R. Dynamic Light Scattering: With Applications to Chemistry, Biology, and Physics. New York: Dover; 2000.

16. Bohren, CF.; Huffman, DR. Absorption and Scattering of Light by Small Particles. New York: Wiley; 1983.

17. Abate Y, et al. Chem. Phys. Lett. 2009; 474:146.

18. Rodríguez-Fernández J, et al. J. Phys. Chem. C. 2007; 111:5020. 


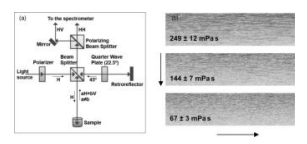

FIG. 1.

(a) PS-OCT interferometer setup. (b) Example $M$-mode images [using an absolute value of $\tilde{a}(z, t)]$ in the $H V$ configuration showing an increasing rate of intensity fluctuations for samples with decreasing viscosities. (Note: Intensity fluctuations only up to $40 \mathrm{~ms}$ shown.) 


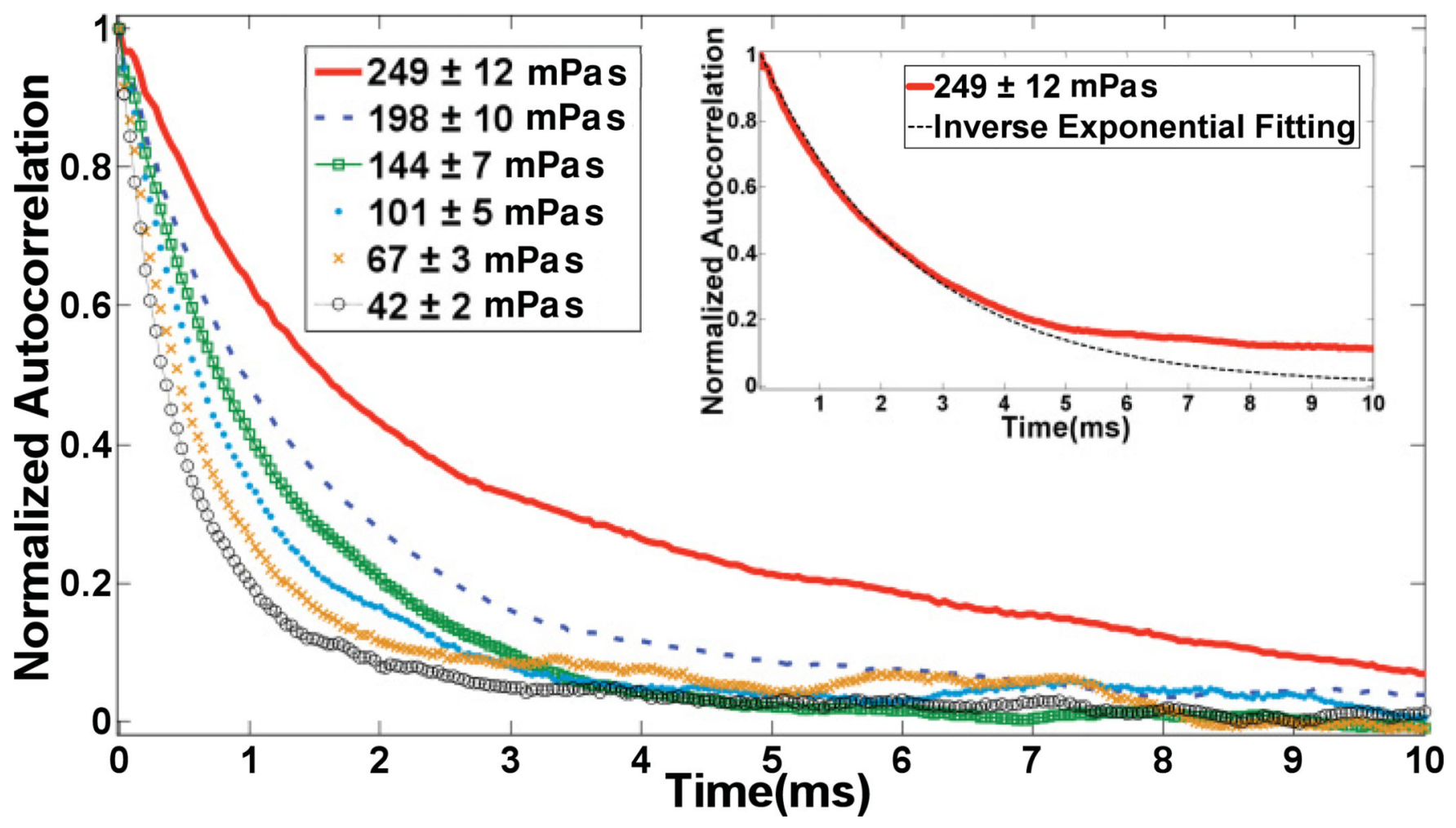

FIG. 2.

(Color online) Representative $g_{H V}^{(1)}(\tau)$ for samples with varying viscosity. Decay time is observed to decrease as the viscosity decreases. The inset shows a representative inverse exponential fitting to $g_{H V}^{(1)}(\tau)$ (dotted line), based on Eq. (1). 


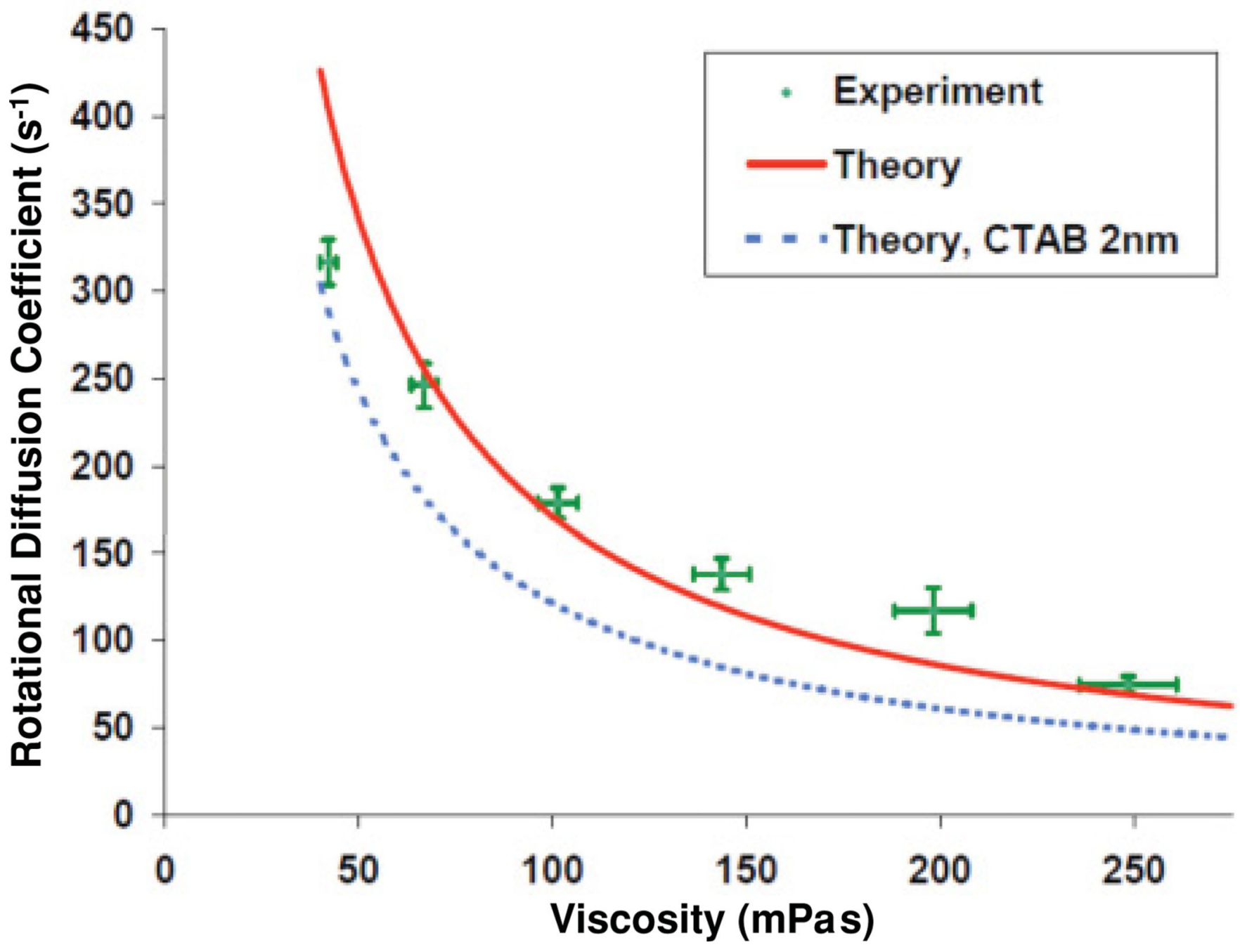

FIG. 3.

(Color online) Comparison of experimental $D_{R}$ with theoretical predictions assuming GNRs as smooth cylinders [14] (solid line: for the actual sizes of the GNRs; dotted line: considering an average CTAB layer of $2 \mathrm{~nm}$ ). 


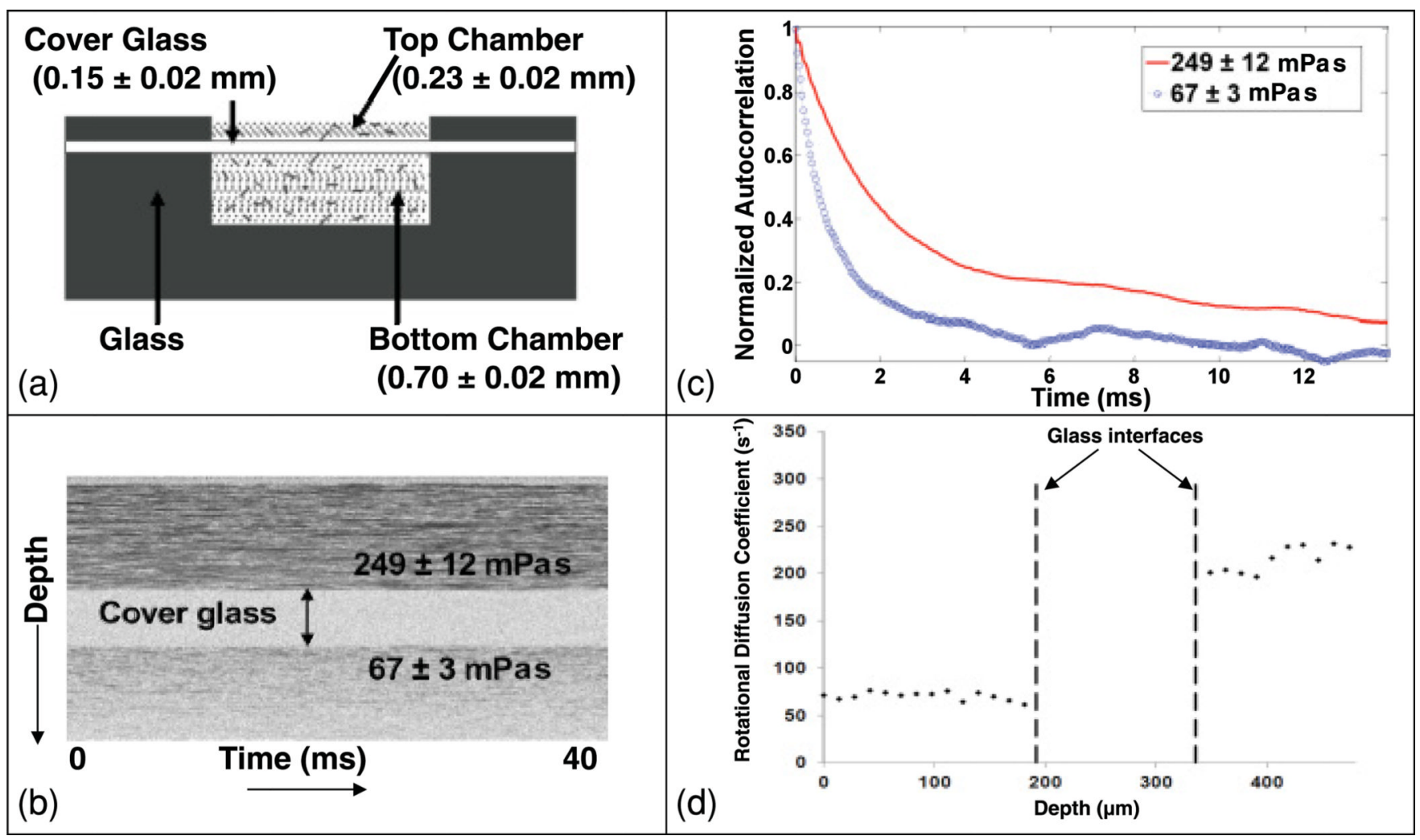

FIG. 4.

(Color online) (a) Doublechamber design. (b) $M$-mode images [using an absolute value of $\tilde{a}(z, t)]$ showing samples with different viscosities separated by a cover glass. (Note: Intensity fluctuations only up to $40 \mathrm{~ms}$ shown.) (c) $g_{H V}^{(1)}(\tau)$ of the samples showing two different decay time scales. (d) $D_{R}$ as a function of depth in the double chamber. 